\title{
COVID Stress Syndrome: Clinical and Nosological Considerations
}

\author{
Steven Taylor ${ }^{1}$ (D) \\ Accepted: 21 January 2021 / Published online: 3 March 2021 \\ (C) The Author(s), under exclusive licence to Springer Science+Business Media, LLC part of Springer Nature 2021
}

\begin{abstract}
Purpose of Review To review the current state of knowledge on the newly proposed COVID Stress Syndrome. Recent Findings The syndrome consists of five inter-correlated elements: (a) fear of SARSCoV2 infection and fear of coming into contact with objects or surfaces contaminated with the coronavirus; (b) fear of socio-economic impacts of the pandemic; (c) fear of foreigners for fear that they are infected; (d) pandemic-related compulsive checking and reassurance-seeking; and (e) pandemic-related traumatic stress symptoms. A severe form of the syndrome, characterized by clinically significant distress and impairment in functioning, is the COVID Stress Disorder, which is regarded as a pandemic-related adjustment disorder. Several treatment options exist but further research is needed.

Summary Research during the COVID-19 pandemic has identified a pandemic-related adjustment disorder. The diagnosis of COVID Stress Syndrome should be made only after ruling out other disorders that could potentially account for the pattern of symptoms, such as obsessive-compulsive disorder and posttraumatic stress disorder. Further studies are needed to investigate the long-term course of the syndrome. Similar adjustment disorders may arise in future pandemics. Accordingly, understanding the COVID Stress Syndrome may facilitate efforts to understand and treat psychopathology in future pandemics.
\end{abstract}

Keywords COVID-19 · COVID Stress Syndrome · COVID Stress Scales $\cdot$ Pandemics $\cdot$ Coronaphobia $\cdot$ Adjustment disorder

\section{Introduction}

It has long been observed that widespread outbreaks of infectious diseases such as pandemics lead to widespread outbreaks of fear [1•]. Until quite recently, these fears were conceptualized narrowly as being something akin to simple mono-phobias. For example, during the Spanish Flu pandemic in 1918, there were descriptions of "flu-phobia" [2-5]. During the Ebola outbreak in West Africa in 2014, when there had been only a single case of Ebola virus disease in the USA, researchers in the USA reported widespread "fearbola" [6]. In other infectious disease outbreaks, researchers documented fears of Zika virus disease, swine flu, and avian flu, all described as mono-phobias or highly specific forms of anxiety $[1 \bullet, 7,8]$. Similarly, during the early months

This article is part of the Topical collection on Anxiety Disorders

Steven Taylor

steven.taylor@ubc.ca

1 Department of Psychiatry, University of British Columbia, 2255 Wesbrook Mall, Vancouver, BC V6T 2A1, Canada of the COVID-19 pandemic, researchers conceptualized and proposed to measure narrowly defined forms of coronavirus anxiety, called "coronaphobia" [9・•].

More recently, clinical investigators began to question the assumption that pandemic-related fears are simple mono-phobias. There were good reasons for questioning the monophobia assumption. Pre-COVID-19 research and case descriptions provided strong hints that pandemic fears might be part of a broader syndrome. Pre-COVID-19 studies showed, for example, that fears of infection were correlated with obsessive-compulsive symptoms such as compulsive checking and reassurance-seeking, and research on previous outbreaks (e.g., SARS, Spanish flu) showed that fears could be associated with posttraumatic stress symptoms, particularly re-experiencing symptoms (e.g., intrusive thoughts, nightmares) [1•].

Given these considerations, my colleagues and I sought to investigate the nature of COVID-19-related fear and distress, as part of our program of research into the psychology of COVID-19. Our goals were to identify and measure important COVID-19-related psychological phenomena, with various practical and long-term goals including the goal of determining whether patterns of COVID-19-related distress were part of some general pandemic distress reaction that could be identified and treated. We sought to investigate whether was there 
a simple mono-phobia reaction, as had been assumed in previous research or was there a broader syndrome. To investigate this issue, we created the COVID Stress Scales [10••].

\section{COVID Stress Syndrome: Structure and Measurement}

The COVID Stress Scales were derived on the basis of previous theory and research $[1 \bullet]$, which indicated that several domains of pandemic-related distress were important to assess the following: (a) Fear of becoming infected with the novel coronavirus; (b) fear of coming into contact with fomites (objects or surfaces) possibly contaminated with the coronavirus; (c) fear of coming into contact with foreigners for fear that they might be carrying the infection (i.e., disease-related xenophobia); (d) fear of the socio-economic consequences of the pandemic (e.g., job loss); (e) compulsive checking and reassurance-seeking regarding possible pandemic-related threats; and (f) traumatic stress symptoms about the pandemic (e.g., nightmares, intrusive thoughts). Replicated factor analytic analyses, based on responses from samples totaling over 7000 people, showed that these six domains loaded on five factors, with the first and second domains (fear of infection and fear of fomites) loading on a common factor $[10 \bullet \cdot, 11]$.

The five COVID Stress Scales have shown good-toexcellent performance on various indices of reliability and validity $[10 \bullet \bullet, 11]$, and have been translated into over a dozen different languages for research purposes (available at www. coronaphobia.org). The study on developing the COVID Stress Scales [10•0] was conceived in January and February, 2020, with data collection taking place during March and April, 2020. During that period, there was widespread media attention about COVID-19 in Canada and the USA, but relatively few cases of infection in those countries. Infection was largely restricted to China. Although it is possible that the time of data collection may have had some impact on the scores on the scales (e.g., the xenophobia scale), the patterns of correlates and factor restructure were replicated in studies in which data was collected at later times during the pandemic and in other countries [e.g., 11].

The test-retest reliability of scales remains to be investigated. The scales were intended as symptom measures, not trait scales, and so temporal stability of scale scores was not a primary concern. It was expected that scores would likely fluctuate to some degree depending on the degree of COVID-19-related threat that the person was experiencing. Indeed, research suggests that COVID-19-related distress has fluctuated over time, with fears rising as the prevalence of infection was increasing, and falling as infection rates were declining [9••].

The five COVID Stress Scales are strongly correlated with one another, with inter-correlations scales ranging from .41 to .73 , which suggests that they form a coherent syndrome called the COVID Stress Syndrome [10••]. Network analyses of the five scales indicated that fear of becoming infected with COVID-19 is at the core of the network [12••].

\section{Demographic and Premorbid Features}

Greater severity of the COVID Stress Syndrome, as assessed by the total score on the COVID Stress Scales, was associated with younger age, female gender, unemployment status, low educational attainment, Asian and Hispanic ethnicities, and a diagnosis of COVID-19 [12••]. Greater severity of scores was also correlated with a pre-existing (pre-COVID-19) diagnosis of a mental health disorder [12••], such as a pre-existing mood or anxiety disorder [13]. Greater scores were correlated with pre-existing health anxiety and greater perceived vulnerability to disease, but not with pre-existing general medical conditions $[10 \bullet, 12 \bullet \cdot]$. Higher scores were associated with greater pre-COVID-19 severity of various traits and symptoms, including disgust sensitivity, anxiety sensitivity, intolerance of uncertainty, obsessive-compulsive checking rituals, and obsessive-compulsive contamination concerns [12••]. In summary, greater severity of the COVID Stress Syndrome was associated with premorbid psychopathology or vulnerability traits and with particular demographic features. The meaning of the demographic findings remains to be further investigated. It is likely that particular demographic groups were exposed to higher levels of particular stressors during the pandemic (e.g., financial stressors, racism), which likely increased the severity of COVID stress symptoms.

\section{Relationship to Other Pandemic-Related Phenomena}

The severity of the COVID Stress Syndrome, as assessed by the total score on the COVID Stress Scales, was associated with high levels of general anxiety and depression during the pandemic, with greater distress during social distancingrelated self-isolation, greater avoidance of public places where COVID-19 might be encountered (e.g., supermarkets), and with greater fear and avoidance of people who might be infected with COVID-19 such as healthcare workers [10••, 14, 15]. Research on fear and avoidance of healthcare workers focused on participants who were not healthcare workers. Other research has focused exclusively on healthcare workers and found that, among these workers, the severity of COVID Stress Syndrome is correlated with the degree of fear that the respondent is an asymptomatic carrier of SARSCoV2 who might inadvertently infect other people [16].

During self-isolation, people with high scores on the COVID Stress Scales, compared to people with lower scores, were more likely to engage in self-defeating forms of 
emotion-focused coping such as over-eating, drug and alcohol abuse, and excessive online shopping [12••]. More generally, regardless of whether or not the person was in self-isolation, the severity of the COVID Stress Syndrome was associated with drug and alcohol abuse [17]. Of the five elements of the COVID Stress Syndrome, the tendency to abuse drugs and alcohol was most strongly linked to the severity of COVID19-related traumatic symptoms, which raises the question of whether people with severe forms of the COVID Stress Syndrome were abusing substances in an effort to dampen symptoms such as nightmares and disturbing intrusive thoughts about the pandemic [17].

To investigate the relationship of the COVID Stress Syndrome with the diverse array of pandemic-related phenomena (e.g., panic buying, use of personal protective equipment), we conducted a comprehensive network analysis [18], using the $\mathrm{R}$ qgraph statistical program [19]. In addition to investigating the COVID Stress Syndrome, we examined a second "syndrome," defined as the COVID Disregard Syndrome. This was not a syndrome in the medical sense of the word, but rather a constellation of behaviors and attitudes that centered around the belief that the COVID-19 threat is exaggerated. The main results of the network analysis are summarized in Fig. 1. The figure shows the links (regularized partial correlations) between nodes in the network (for all links, $p<.003)$. The magnitude of the strength of connections among nodes is indicated by shorter, thicker lines, with positive associations in green and negative associations in red. As expected, the elements of the COVID Stress Syndrome (blue ellipses) were tightly linked together, as were the elements of the COVID Disregard Syndrome (yellow ellipses). The COVID Disregard Syndrome was negatively associated with hand and cough hygiene adherence and with the COVID Stress Syndrome. The COVID Stress Syndrome was associated with a range of pandemic-related phenomena, including avoidance of supermarkets and drug stores (for fear of infection), fear and avoidance of healthcare workers (also for fear of infection), use of personal protective equipment (masks, gloves, and visors), and panic buying and stockpiling of supplies in preparation for self-isolation. The COVID Stress Syndrome was also linked, to a lesser extent, with belief in COVID-19 conspiracy theories (e.g., belief that the novel coronavirus was engineered as a bioweapon) and with COVID19-related anti-vaccination attitudes (e.g., worry about unforeseen harmful effects of the vaccine). In summary, the result of the network analysis indicated that the COVID Stress Syndrome is linked to many different types of pandemicrelated phenomena, particularly those associated with fear, avoidance, and self-protective behaviors.

\section{Diagnostic Considerations}

Research using the COVID Stress Scales clearly and consistently shows that COVID-related distress is not simply some

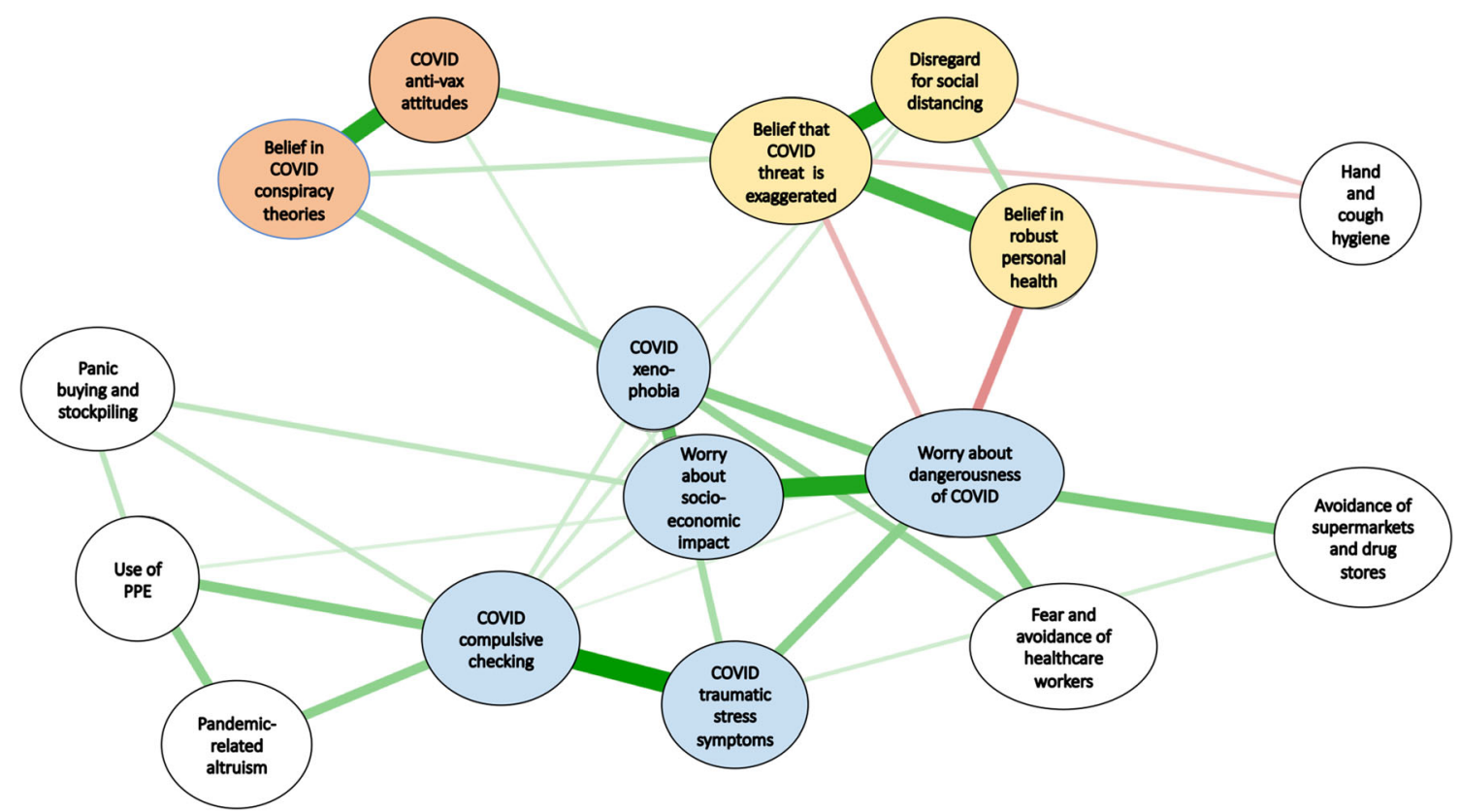

Fig. 1 Network analysis: strength of interconnections (regularized partial correlations) among the elements in the network (green = positive and red = negative connections). Stronger connections are indicated by shorter and thicker lines. PPE = personal protective equipment. Reprinted from Taylor S, Paluszek M, Landry C, Rachor GS, Asmundson GJG. Worry, avoidance, and coping during the COVID-19 pandemic: A comprehensive network analysis. Journal of Anxiety Disorders. 2020:102327. https://doi.org/10.1016/j.janxdis.2020.102327. Copyright (2020), with permission from Elsevier 
form of mono-phobia. The various fears and other symptoms associated with COVID-19 form a coherent COVID Stress Syndrome. Latent class analyses of the COVID Stress Scales suggest that the COVID Stress Syndrome is essentially dimensional in nature rather than having a simple "disorder" versus "non-disorder" structure [12••]. This is not surprising given that many, if not most, forms of psychopathology are dimensional rather than categorical in nature [20-24]. Unfortunately, however, the dimensional nature of the COVID Stress Syndrome raises problems for clinical practice and for the allocation of clinical resources; if the syndrome is dimensional, then how do we identify people in greatest need of clinical services?

We considered several approaches for addressing the problem. Cut-off scores could be developed, using some arbitrary criterion (e.g., particular scale scores or total score). A problem with this approach is that mean scores on the scales have changed over the course of the COVID-19 pandemic; for example, rising and falling in response to the degree of threat (infection rate) occurs in the respondents' community [9••]. It might not be possible to derive cut-off scores on the COVID Stress Scales in an absolute sense, but cut-offs can be derived based on how people in a given population respond at a given time (e.g., scores above the 90th percentile, assessed at a given time). Such an approach is justifiable if the COVID Stress Syndrome is conceptualized as an adjustment reaction (as discussed below), characterized by an excessive amount of distress in response to a given stressor. In other words, a COVID Stress Disorder could be diagnosed in people who have developed unusually intense distress, as compared to other people who have encountered the same stressor.

An additional, complementary approach, borrowed from the psychiatric nomenclature $[25,26]$ is to use both distress and impairment in functioning to identify cases in which the COVID Stress Syndrome could be regarded as constituting a clinically significant disorder; that is, a COVID Stress Disorder. Current approaches to diagnosing mental disorders, such as ICD-11 and DSM-5, require distress and/or impairment to diagnose mental disorders. A problem in diagnosing impairment during the COVID-19 pandemic is that a person might have impairment due to restrictions placed on his or her social and occupational functioning due to social distancing restrictions (e.g., lockdown, closure of places of work). Accordingly, in our research on the COVID Stress Disorder, we measured impairment as impairment in social or occupational functioning specifically due to one's distress about COVID-19; for example, being unable to fulfill one's occupational role due to severe COVID-19-related anxiety.

Along with the COVID Stress Scales (measuring distress), participants in our research completed a scale in which they were asked to rate the degree of functional impairment (social or occupational) specifically due to distress about COVID-19 [9••]. This measure was an adaptation of the Sheehan Disability Scale [27]. Participants having severe impairment due to COVID-19-related distress (13\% of 2078 adults from the US and Canada) were classified as having a COVID Stress Disorder [9••]. Not surprisingly, scores on the COVID Stress Scales were significantly higher in people classified as having COVID Stress Disorder compared to people who did not meet

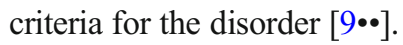

Further research is needed to determine how both scores on the COVID Stress Scales (measuring distress) and scores on our adapted Sheehan Disability Scale (measuring impairment due to COVID-19 distress) can be optimally combined to diagnose COVID Stress Disorder. At the present time, the following working criteria are under evaluation for diagnosing the COVID Stress Disorder. The disorder is diagnosed with either (a) or (b) are present, along with (c): (a) Severe COVID19-related distress, (b) severe impairment in social or occupational functioning specifically due to COVID-19-related distress, and (c) the pattern of distress and impairment is not better explained by some other disorder.

\section{Conceptualizing the COVID Stress Disorder as an Adjustment Disorder}

Although research shows that scores on the COVID Stress Scales are correlated with pre-existing mental health problems, many people with high scores do not have a history of these disorders. For example, of the 268 (13\%) of the 2078 adults who were diagnosed with COVID Stress Disorder, only $26 \%$ of these 268 individuals had a pre-COVID-19 mental disorder [9••]. It is also noteworthy that most $(74 \%)$ people with COVID Stress Disorder did not have a pre-COVID-19 disorder, even if they did tend to have high scores on personality traits (e.g., intolerance of uncertainty) that would predispose them to experiencing intense emotional distress in responses to stressors such as a pandemic, which entails a great deal of stressful uncertainty [1•]. The question arises about how to best classify pandemic-related stress reactions such as the COVID Stress Disorder.

The features of the COVID Stress Disorder overlap with several anxiety-related disorders, such as obsessivecompulsive disorder, specific phobia, illness anxiety disorder, and posttraumatic stress disorder [25, 26]. However, the symptoms of the COVID Stress Disorder do not neatly fit into any one of these categories. COVID Stress Disorder is better conceptualized as a pandemic-related adjustment disorder. An adjustment disorder, as conceptualized in DSM-5 and ICD11 , is characterized by clinically significant (i.e., markedly distressing and/or impairing) emotional and behavioral symptoms arising in response to a life stressor that are not due to some other disorder and may be either acute or chronic. To illustrate, the ICD-11 definition of adjustment disorder captures, in a general sense, many of the features of the COVID Stress Disorder: 
Adjustment disorder is a maladaptive reaction to an identifiable psychosocial stressor or multiple stressors (e.g. divorce, illness or disability, socio-economic problems, conflicts at home or work) that usually emerges within a month of the stressor. The disorder is characterised by preoccupation with the stressor or its consequences, including excessive worry, recurrent and distressing thoughts about the stressor, or constant rumination about its implications, as well as by failure to adapt to the stressor that causes significant impairment in personal, family, social, educational, occupational or other important areas of functioning. The symptoms are not better explained by another mental disorder (e.g., Mood Disorder, another Disorder Specifically Associated with Stress) and typically resolve within 6 months, unless the stressor persists for a longer duration. (https://icd.who.int/browse11/1-m/en\#/http://id.who.int/ icd/entity/264310751, accessed December 12, 2020)

Conceptualizing the COVID Stress Disorder as a pandemic-related adjustment reaction does not imply that the disorder is trivial. Adjustment disorders meet the impairment and distress criteria for mental disorders in general [25] and merit serious attention from treating clinicians $[28,29]$. Although it may be that many cases of adjustment disorder abate once the stressor has passed, this is not invariably the case; some cases become chronic, with progressively intensifying psychopathology $[29,30]$. Important unanswered questions concern the prevalence and chronicity of the COVID Stress Disorder. That is, what proportion of people are likely to have acute (e.g., $<6$ month) reactions versus chronic symptoms? A further question concerns the best way of predicting who is likely to have acute or chronic symptoms.

\section{Treatment Implications}

Among the major challenges during the COVID-19 pandemic concerns the need to provide mental health services to large numbers of people in a way that limits or obviates face-to-face contact. Online (E-health) psychological services have rapidly arisen in many parts of the world to address this problem [31]. For pandemic-related distress in general, options include online self-help psychological exercises, psychoeducation through Internet platforms and mobile phone applications, and telephone or videoconference meetings with mental health professionals. These can either be stand-alone or integrated with one another, and also have the potential for use in a stepped care fashion, for example, starting with the least intensive intervention (e.g., self-help) and moving toward more intensive (e.g., clinician-implemented) as necessary.

Online and telehealth interventions show promise [31], but they have yet to be evaluated specifically for the COVID
Stress Syndrome. Existing online cognitive-behavioral selfhelp and clinician-administered interventions [32] could be adapted to treat many aspects of the COVID Stress Syndrome, such as COVID-19-related fears. Given that the fear of COVID-19 infection is at the core of the COVID Stress syndrome, this fear could be targeted by cognitivebehavioral anxiety management methods, such as exercises involving cognitive structuring, worry management, and stress management. Treating the core element of the syndrome may have beneficial effects on other elements of the syndrome. However, if treating the fear of infection does not have a sufficient impact on other elements of the syndrome, then additional interventions could be implemented.

COVID-19-related compulsive checking and reassuranceseeking could be directly targeted by online adaptations of cognitive-behavioral methods for treating health anxiety [33]. If obsessive-compulsive symptoms are severe and refractory, there is debate as to how they should be best treated during the COVID-19 pandemic, particularly contamination fears and washing compulsions. Some clinicians have suggested that for such patients, it is simpler and probably safer to implement pharmacotherapy for obsessive-compulsive symptoms, rather than encourage patients to neglect handwashing guidelines, which might be required as part of exposure and response prevention interventions [34]. Clinicians advocating for exposure and response prevention have proposed guidelines for conducting exposure in the context of COVID-19 [35, 36]. Combined exposure therapy and pharmacotherapy is also an option.

With regard to the traumatic stress symptoms associated with the COVID Stress Syndrome, these symptoms may consist of intrusive thoughts and images of news media or social media stories about the pandemic. In such cases, patients could be advised to limit their exposure to disturbing media, that is, staying informed from reliable news services but not spending hours per day watching disturbing (and possibly exaggerated) social media or news media accounts of traumatic pandemic-related events. If this strategy is insufficient, then pharmacotherapy or cognitive-behavioral treatments for posttraumatic stress disorder could be considered. For example, trauma-related exposure therapy can be successfully implemented via telehealth by suitably trained clinicians $[37,38]$.

\section{Conclusions}

Until very recently, pandemic-related fears were considered to be mono-phobias; that is, simple, excessive fear reactions. More recently, research shows that these fears are part of a broader syndrome, which can be conceptualized as a pandemic-related adjustment disorder. During COVID-19, research provides evidence of a COVID Stress Syndrome, which in its severe form is an adjustment disorder called the 
COVID Stress Disorder. In the months since the syndrome and disorder were recognized, a great deal of research has been conducted to further understand the nature and correlates of COVID-19-related distress. Future research is required to better understand the time course and optimal treatment of the syndrome and disorder. Adjustment disorders are not trivial conditions; they meet all the requirements of distress and impairment for mental disorders in general. A major assumption with adjustment disorders is that the psychopathology will abate in most cases once the stressor abates. With regard to the COVID-19 pandemic, which was ongoing at the time this article was written, future research is needed to determine the extent to which the COVID Stress Syndrome (and Disorder) abate once the pandemic is over. Some cases may become chronic, although this remains to be investigated. But even if most cases abate once the pandemic is over, there is still a need to treat highly distressed people during the course of the pandemic. Research is also needed to evaluate the extent to which currently available mental health resources are beneficial in treating the COVID Stress Syndrome and Disorder. Further research is needed to firmly establish the boundaries of the syndrome (e.g., are there other core features that need to be included?) and to investigate whether the syndrome substantially varies across cultures and demographic groups. Additional measures also need to be developed and evaluated. Currently, the assessment of the COVID Stress Syndrome and Disorder is limited to self-report measures. Clinicianadministered interview methods could be developed to further evaluate the nature of COVID-19-related distress.

The diagnosis of COVID Stress Syndrome should be made only after ruling out other disorders that could potentially account for the pattern of symptoms, such as obsessive-compulsive disorder and posttraumatic stress disorder. Further studies are needed to investigate the long-term course of the syndrome. The COVID Stress Disorder is conceptualized as a pandemicrelated adjustment disorder. Similar adjustment disorders will likely arise in future pandemics. Accordingly, understanding the COVID Stress Syndrome and Disorder may facilitate efforts to understand and treat psychopathology in future pandemics.

Funding This article did not receive any specific grant from funding agencies in the public, commercial, or not-for-profit sectors.

\section{Declarations}

Conflict of Interest There are no conflicts of interest associated with this paper.

Human and Animal Rights and Informed Consent All reported studies/ experiments with human or animal subjects performed by the author and cited in this article have complied with all applicable ethical standards (including the Helsinki declaration and its amendments, institutional/national research committee standards, and international/national/institutional guidelines).

\section{References}

Papers of particular interest, published recently, have been highlighted as:

- Of importance

- Of major importance

1. Taylor S. The psychology of pandemics: preparing for the next global outbreak of infectious disease. Newcastle upon Tyne: Cambridge Scholars Publishing; 2019. Published a few weeks before the outbreak of COVID-19, this book provided the first comprehensive review and analysis of the role of psychological factors in pandemics.

2. Evening Express (Los Angeles). Watch out or fluphobia'll catch yuh! p. 3. 1918, October 26.

3. Spokesman Review (Washington State). Cause and treatment of fluphobia. 1918, October 18:p. 4.

4. Sandusky Register (Ohio). Fear of Spanish "flu" fills folks with dread. 1918, October 18:p. 6.

5. Baltimore Sun (Maryland). Flee to hills from "flu". p. 2. 1918, November 6.

6. Blakey SM, Reuman L, Jacoby RJ, Abramowitz JS. Tracing "fearbola": psychological predictors of anxious responding to the threat of Ebola. Cognit Ther Res. 2015;39:816-25. https://doi.org/ 10.1007/s10608-015-9701-9.

7. Blakey SM, Abramowitz JS. Psychological predictors of health anxiety in response to the Zika virus. J Clin Psychol Med Settings. 2017;24:270-8. https://doi.org/10.1007/s10880-0179514-y.

8. Wheaton MG, Abramowitz JS, Berman NC, Fabricant LE, Olatunji BO. Psychological predictors of anxiety in response to the H1N1 (swine flu) pandemic. Cognit Ther Res. 2012;36:210-8. https://doi. org/10.1007/s10608-011-9353-3.

9.• Asmundson GJG, Taylor S. Coronaphobia revisited: A state-of-theart on pandemic-related fear, anxiety, and stress. J Anxiety Disord. 2020;76:102326. https://doi.org/10.1016/j.janxdis.2020.102326 Introduced the concept of the COVID Stress Disorder.

10.• Taylor S, Landry CA, Paluszek MM, Fergus TA, McKay D, Asmundson GJG. Development and initial validation of the COVID Stress Scales. J Anxiety Disord. 2020;72:102232. https:// doi.org/10.1016/j.janxdis.2020.102232 Reported the development and validation of the COVID Stress Scales.

11. Khosravani V, Asmundson GJG, Taylor S. The Persian COVID Stress Scales (Persian-CSS) and COVID-19-related stress reactions in patients with obsessive-compulsive and anxiety disorders. J Obsessive Compuls Relat Disord. 2021;28:100615.

12.• Taylor S, Landry CA, Paluszek MM, Fergus TA, McKay D, Asmundson GJG. Covid stress syndrome: concept, structure, and correlates. Depress Anxiety. 2020;37:706-14. https://doi.org/10. 1002/da.23071 Introduced the concept of the COVID Stress Syndrome.

13. Asmundson GJG, Paluszek MM, Landry CA, Rachor GS, McKay $\mathrm{D}$, Taylor S. Do pre-existing anxiety-related and mood disorders differentially impact COVID-19 stress responses and coping? J Anxiety Disord. 2020;74:102271. https://doi.org/10.1016/j. janxdis.2020.102271.

14. Taylor S, Landry CA, Rachor GS, Paluszek MM, Asmundson GJG. Fear and avoidance of healthcare workers: an important, underrecognized form of stigmatization during the COVID-19 pandemic. J Anxiety Disord. 2020;75:102289. https://doi.org/10.1016/j. janxdis.2020.102289.

15. Taylor S, Landry CA, Paluszek MM, Asmundson GJG. Reactions to COVID-19: differential predictors of distress, avoidance, and 
disregard for social distancing. J Affect Disord. 2020;277:94-8. https://doi.org/10.1016/j.jad.2020.08.002.

16. Delgado-Gallegos JL, Montemayor-Garza RJ, Padilla-Rivas GR, Franco-Villareal H, Islas JF. Prevalence of stress in healthcare professionals during the COVID-19 pandemic in northeast Mexico: a remote, fast survey evaluation, using an adapted COVID-19 Stress Scales. Int J Environ Res Public Health. 2020;17. https://doi.org/10. 3390/ijerph17207624.

17. Taylor S, Paluszek M, Rachor GS, McKay D, Asmundson GJG. Substance use and abuse, COVID-19-related distress, and disregard for social distancing: a network analysis. Addict Behav. 2020;114: 106754.

18. Taylor S, Paluszek M, Landry C, Rachor GS, Asmundson GJG. Worry, avoidance, and coping during the COVID-19 pandemic: a comprehensive network analysis. J Anxiety Disord. 2020:102327. https://doi.org/10.1016/j.janxdis.2020.102327.

19. Epskamp S, Cramer AO, Waldorp LJ, Schmittmann VD, Borsboom D. Qgraph: Network visualizations of relationships in psychometric data. J Stat Softw. 2012;48:1-18. https://doi.org/10. 18637/jss.v048.i04.

20. Haslam N, Holland E, Kuppens P. Categories versus dimensions in personality and psychopathology: a quantitative review of taxometric research. Psychol Med. 2012;42:903-20. https://doi. org/10.1017/S0033291711001966.

21. Kramer MD, Arbisi PA, Thuras PD, Krueger RF, Erbes CR, Polusny MA. The class-dimensional structure of PTSD before and after deployment to Iraq: evidence from direct comparison of dimensional, categorical, and hybrid models. J Anxiety Disord. 2016;39:1-9. https://doi.org/10.1016/j.janxdis.2016.02.004.

22. Ofrat S, Krueger RF, Clark LA. Dimensional and model-based alternatives to categorical personality disorders. In: Blaney $\mathrm{PH}$, Krueger RF, Millon T, editors. Oxford textbook of psychopathology. 3rd ed. New York: Oxford University Press; 2015. p. 617-38.

23. Walton KE, Ormel J, Krueger RF. The dimensional nature of externalizing behaviors in adolescence: evidence from a direct comparison of categorical, dimensional, and hybrid models. J Abnorm Child Psychol. 2011;39:553-61. https://doi.org/10.1007/s10802010-9478-y.

24. Widiger TA, Gore WL. Mental disorders as discrete clinical conditions: dimensional versus categorical classification. In: Beidel DC, Frueh BC, Hersen M, editors. Adult psychopathology and diagnosis. 7th ed. Hoboken: Wiley; 2014. p. 3-33.

25. American Psychiatric Association. Diagnostic and statistical manual of mental disorders. 5th ed. Arlington: Author; 2013.

26. World Health Organization. International classification of diseases. 11th edth ed., 2018. https://icd.who.int/en. Accessed 5 Jan 2021

27. Sheehan DV. The anxiety disease. New York: Scribners; 1983.

28. Kazlauskas E, Quero S. Adjustment and coronavirus: how to prepare for COVID-19 pandemic-related adjustment disorder worldwide? Psychol Trauma. 2020;12(S1):S22-S4. https://doi. org/10.1037/tra0000706.

29. Bachem R, Casey P. Adjustment disorder: a diagnosis whose time has come. J Affect Disord. 2018;227:243-53. https://doi.org/10. 1016/j.jad.2017.10.034.

30. O'Donnell ML, Alkemade N, Creamer M, McFarlane AC, Silove D, Bryant RA, et al. A longitudinal study of adjustment disorder after trauma exposure. Am J Psychiatry. 2016;173:1231-8. https:// doi.org/10.1176/appi.ajp.2016.16010071.

31. Yue J-L, Yan W, Sun Y-K, Yuan K, Su S-Z, Han Y, et al. Mental health services for infectious disease outbreaks including COVID19: a rapid systematic review. Psychol Med. 2020;50:2498-513. https://doi.org/10.1017/S0033291720003888.

32. Varker T, Brand RM, Ward J, Terhaag S, Phelps A. Efficacy of synchronous telepsychology interventions for people with anxiety, depression, posttraumatic stress disorder, and adjustment disorder: a rapid evidence assessment. Psychol Serv. 2019;16:621-35. https://doi.org/10.1037/ser0000239.

33. Taylor S, Asmundson GJG. Treating health anxiety. New York: Guilford; 2004

34. Fineberg NA, Van Ameringen M, Drummond L, Hollander E, Stein DJ, Geller D, et al. How to manage obsessive-compulsive disorder (OCD) under COVID-19: a clinician's guide from the International College of Obsessive Compulsive Spectrum Disorders (ICOCS) and the Obsessive-Compulsive and Related Disorders Research Network (OCRN) of the European College of Neuropsychopharmacology. Compr Psychiatry. 2020;100: 152174. https://doi.org/10.1016/j.comppsych.2020.152174.

35. Shafran R, Coughtrey A, Whittal M. Recognising and addressing the impact of COVID-19 on obsessive-compulsive disorder. Lancet Psychiatry. 2020;7:570-2. https://doi.org/10.1016/s2215-0366(20) 30222-4.

36. Jassi A, Shahriyarmolki K, Taylor T, Peile L, Challacombe F, Clark B, et al. OCD and COVID-19: a new frontier. Cogn Beh Therap. 2020;13. https://doi.org/10.1017/S1754470X20000318.

37. Fina BA, Wright EC, Rauch SAM, Norman SB, Acierno R, Cuccurullo L-AJ, et al. Conducting prolonged exposure for PTSD during the COVID-19 pandemic: considerations for treatment. Cogn Behav Pract. 2020. https://doi.org/10.1016/j.cbpra.2020.09. 003.

38. Wells SY, Morland LA, Wilhite ER, Grubbs KM, Rauch SAM, Acierno R, et al. Delivering prolonged exposure therapy via videoconferencing during the COVID-19 pandemic: an overview of the research and special considerations for providers. J Trauma Stress. 2020;33:380-90. https://doi.org/10.1002/jts.22573.

Publisher's Note Springer Nature remains neutral with regard to jurisdictional claims in published maps and institutional affiliations. 